\title{
Underwater Recording and 3D Modeling during a Dive Training Course: A Case Study at Panormos, Skopelos,
} Greece

\author{
Kimon Papadimitriou, Anastasios Ktistis and Ioanna Sarakinou \\ Department of Cadastre, Photogrammetry and Cartography, School of Rural and Surveying Engineering, Faculty of Engineering, \\ Aristotle University of Thessaloniki, Thessaloniki 54124, Greece
}

\begin{abstract}
In the field of underwater survey, there is a variety of methods which differ from each other in terms of the operating environment and the purpose that each method is used. Hence, some of the state-of-the-art methods, that have many applications in the various scientific fields nowadays, are introduced in this paper. Additionally, the application of the procedures for an underwater survey in shallow depths is analyzed in accordance with the training standards of the PADI Underwater Survey Diver distinctive specialty. The main goal is to demonstrate not only the theoretical background of underwater surveys but also their operational issues, in order to facilitate the development of knowledge and skills during a training course. Finally, a case study for the recording and 3D modeling of the seabeds' morphology in shallow water is presented as it has been organized and accomplished by the participants of such a training course. By this way, it is expected that the reader will understand explicitly the application of the procedures prior, during and after the dive for an underwater survey in shallow depths.
\end{abstract}

Key words: Underwater survey, training course, photogrammetry, 3D modeling.

\section{Introduction}

In several cases of research (by geologists, biologists, archaeologists and engineers etc.), the mapping of underwater natural and cultural features is essential for documentation, presentation, spatio-temporal analysis or monitoring purposes. In the field of underwater surveying, there is a variety of methods which differ from each other in terms of the operating environment and the purpose for which each method is used. Last spring, International Society for Photogrammetry and Remote Sensing (ISPRS) published the proceedings of the workshop for underwater 3D recording and modeling [1]. Main focus of this event was to bring together scientists, developers and advanced users in underwater 3D recording, and related disciplines for the documentation, in various underwater scenarios

Corresponding author: Kimon Papadimitriou, laboratory training staff, research fields: underwater surveying and environmental mapping. E-mail: paki@auth.gr. related to data processing and 3D modeling [2]. This edition presents some of the state-of-the-art methods that have many applications in the fields of cultural heritage and marine biology.

Many countries have a remarkable underwater cultural heritage. It is very important underwater archaeological sites to be 3D recorded. Some of the goals of an underwater survey of an archaeological site are to generate 3D models and support the underwater excavations. Hence, underwater survey methods play a cardinal role in marine archaeology [3].

Data on marine biological communities conventionally have been obtained by underwater photogrammetry. With these techniques, scientists can observe any change that takes place in a marine ecosystem with the passage of time. Also, thanks to underwater survey marine, biologists are able to study the effects of pollution on selected communities. In conclusion, underwater survey is a useful tool which 
carries out many marine biological surveys [3].

Such kinds of underwater surveys in shallow water include on-site diving [4-12] which requires proper training. The design, the planning and the accomplishment of a dive for the recording of marine features combines a wide body of knowledge and skills beyond the basic ones that are needed for a recreational diving. Additionally, the quality of aquired data is affected by (a) the application of mapping methodologies, (b) the appropriate use of special equipment and (c) the coordination of a diving team before, during and after the underwater survey. In order to cope with the above issues, a training course for the qualification of divers which are going to participate in underwater surveys has been developed. This course has been recently recognized by the Professional Association of Diving Instructors (PADI) as a distinctive specialty. That is, following the specified course outline [13], the participants may get a worthwhile certification that occasionally may be acknowledged by international educational and vocational training authorities [14]. The purpose of the PADI Underwater Survey Diver distinctive specialty course is to provide the basic knowledge and the skills for those divers and researchers with a special interest in natural and cultural features of the marine environment, so, they can be able to participate in the preparation and the carrying out of an underwater survey campaign. The PADI Underwater Survey Diver distinctive specialty course consists of three basic sections and one optional workshop for the processing of the captured data. The first one relates to the knowledge development (theory), the second one to the development of diving skills (practice) and the third one to the application of knowledge and skills under open water conditions.

The knowledge development section of the PADI Underwater Survey Diver distinctive specialty course introduces the participants in the basic concepts of mapping and photogrammetry that apply in underwater surveys, whilst the skills development section familiarizes the divers with the implementation of underwater surveying procedures for the measurements and the $3 \mathrm{D}$ recording in confined water conditions. Both sections include two modules each, one for mapping (related to the measurements) and another for photogrammetry (related to 3D recording). By the end of those modules that are related to the measurements, participants have been able to: (a) set up a grid and sketch its content, (b) measure the depths and calculate the depth difference between points, (c) measure slope or horizontal distances, (d) keep notes for the measurements underwater and (e) write a report for the underwater survey dive. Supplementary, by the end of the modules that are related to 3D recording, participants have been able to take two stripes of at least 4 almost vertical overlapping frames each, following a U-pattern with approximately more than 30\% overlapping between the stripes and $60 \%$ between the sequential frames. This combination of knowledge and skills has provided the background for the planning and the implementation of the underwater survey towards the 3D modeling of the study area.

By this way, participants are becoming able to plan and implement an underwater survey. Although post-diving procedures for the processing of the surveyed data towards the creation of 3D models are beyond the purpose of this course, they are also included as an optional module at those with a special interest on such kind of methods, techniques and their applications.

As a result, the compliance with the above mentioned procedures establishes a protocol for the recording of underwater features so that the resulting data can then be used towards the 3D modeling.

This paper presents the application of the proposed protocol, during a training course of the PADI Underwater Survey Diver distinctive specialty, towards the 3D modeling of a small part at a diving site. The aim of this paper is to present the results from the application of the knowledge and the skills 
that have been developed during this course. The preparation and the implementation of an underwater survey, have been performed by the participants of the course, in confined water conditions. The objectives of this work include: (a) the description of the underwater survey procedures which have been followed by the participants before, during and after the dives, (b) the data processing for the creation of a 3D model for the study area and (c) an accuracy estimation of the produced model.

\section{Case Study and Materials}

The course outline for the PADI Underwater Survey Diver distinctive specialty is considered the general protocol for the planning and the implementation of the presented case study. The underwater survey has been carried out by five participants and one trainer during a diving session of the course, in confined water conditions. The data has been processed during a workshop that followed the dive and in order to produce the 3D model. All of the participants have had some previous experience in mapping projects.

\subsection{Study Area}

The training has been conducted on October 2015 at Adrina beach, Panormos, in Skopelos, Greece. The specific beach lies about $100 \mathrm{~m}$ from WNW to ESE direction, accommodates the docking facilities of a nearby hotel and an entry/exit ramp for the swimmers/divers, while it remains sheltered from the dominant north winds. The seabed is mostly gravel with some sandy parts and small pieces of rocks. The relief is gentle with smooth inclination near the coast (up to $10 \mathrm{~m}$ of depth) which is becoming steeper at a distance of about $50 \mathrm{~m}$ from the coastline to increase up to $30 \mathrm{~m}$ of depth after about $100 \mathrm{~m}$ off shore. The area accommodates a variety of Mediterranean sea life (mostly coastal species and occasionally pelagic ones) and the shallow zone consists a site with confined water conditions for dive training activities.
The survey area extends approximately $3 \mathrm{~m}$ (on the north to south axis) by $7 \mathrm{~m}$ (on the east to west axis) with a mean depth of $3.5 \mathrm{~m}$. The survey has been performed in one day with light clouds and calm sea without current. Underwater visibility was $15 \mathrm{~m}$ and the sea temperature at the surface and during the dive was $23^{\circ} \mathrm{C}$.

\subsection{Equipment}

Apart of the personal scuba equipment for each diver, as outlined in the PADI instructor manual [15], it has been used the following material during the survey: (a) eight steel poles, $150 \mathrm{~cm}$ each, marked with colored tape at the top end, for the marking of the study area, (b) two sledgehammers, $1 \mathrm{~kg}$ each, for putting the poles in place, (c) two retractable reels, 30 $\mathrm{m}$ long each, for the marking of the study area, (d) one Delayed Surface Marking Buoy (DSMB), for the marking of the study area, (e) two blank slates, $23 \mathrm{~cm}$ $\times 31 \mathrm{~cm}$ each, with two pencils, for taking notes, sketching and as a reference for the evaluation of the 3D model, (f) one plastic measuring tape, $20 \mathrm{~m}$ long, (g) two folding plastic rulers, $2 \mathrm{~m}$ each, for the scaling and the evaluation of the 3D model, (h) one carpenter's square, $70 \mathrm{~cm} \times 30 \mathrm{~cm}$, for the evaluation of the 3D model, (i) one metallic plummet tied with $1.5 \mathrm{~m}$ line and a small floater, as a vertical reference for the placement of the poles and the measurements and (j) two underwater mesh bags for carrying. Two underwater action cameras has been used for the photogrammetric coverage of the area: (a) one Gopro Hero 3 Black Edition (focal length of $2.77 \mathrm{~mm}, 35$ $\mathrm{mm}$ camera equivalent with focal length $15 \mathrm{~mm}$, and resolution of 4,000 $\times 3,000$ pixels) and (b) one Rollei $7 \mathrm{~S}$ (focal length $5 \mathrm{~mm}, 35 \mathrm{~mm}$ camera equivalent with focal length $33 \mathrm{~mm}$, and resolution of 4,000 $\times 3,000$ pixels). For the data processing, a desktop PC has been used, operating on Windows 8 (64 bit) with $2 \mathrm{X}$ Intel Xeon E5-2620 V3/2.40 GHz CPUs, 32 GB of RAM and NVIDIA GeForce GTX 970 and running Agisoft Photoscan Pro 1.1.6 (64 bit). 


\subsection{Data}

The images from the two cameras have formed two data sets: the first one with twenty images (recorded with the Gopro Hero 3, aperture f2.8, shutter speed $1 / 120 \mathrm{sec}$, ISO 120-157) and the second one with ninety six images (recorded with the Rollei 7S, aperture f2.7, shutter speed 1/100 sec-1/50 sec, ISO 73-140). Additionally, one folding plastic ruler has been deployed at right angle, with lengths of $80 \mathrm{~cm}$ and $20 \mathrm{~cm}$ on two perpendicular parts and one carpenter's square $(70 \mathrm{~cm}$ by $30 \mathrm{~cm}$ ) have been used as a reference for the scaling the model. Another folding plastic ruler has been deployed at right angle (with equal lengths on the two perpendicular parts) and one blank slate (measured $23 \mathrm{~cm} \times 31 \mathrm{~cm}$ ) has been used as samples for the accurancy estimation of the produced 3D model.

\section{Underwater Survey Procedures}

After the skill development section and before the open water training, it has been included a "minidive" session, in confined water, for an underwater survey and in order to apply the knowledge and the skills that already have been developed by the participants. During this optional part of the training, participants have had the chance to operate as a diving team and to test themselves by adopting a realistic scenario. That is, a "minidive" includes the complete sequence of the procedures toward the 3D modeling of a study area, without the safety and logistic considerations of an open water dive. The data for the presented case study has been recorded during this dive, following the procedures that are described in the following three sections.

\subsection{Pre-dive Procedures}

Pre-dive procedures include the dive briefing and the planning for the site preparation, the measurements and the $3 \mathrm{~d}$ recording. During the dive briefing have been presented and explained: (a) an evaluation of the conditions, (b) the facilities at the dive site, (c) the entry and exit techniques to be used (depending the location and the equipment), (d) the composition of the sea bed and the topography of the site, (e) the depth range on bottom, (f) the ending tank pressure (when to terminate the dive) and (g) any interesting and helpful facts about the site. During the planning for the site preparation have been defined, the tasks for the recognition, the marking and the sketch up of the area. During the planning for the measurements have been defined, the tasks for the measuring of depths and horizontal distances within the area. During the planning for the 3D recording have been defined, the tasks for photographic/video coverage of the area. Finally, the pre-dive procedures have included the preparation of special equipment, the delegation of underwater tasks, a review for underwater communication, gas/depth/time limitations, entries and exits.

\subsection{Dive Procedures}

After the preparation of personal scuba equipment, the suiting up and the planning for the dive have been formulated three teams of divers, one for the site preparation, a second one for the measurements and another for the 3D recording. For each team, a pre-dive safety check and a buoyancy check have been performed before leaving the surface. Sequentially, three teams have dived (for site preparation, measurements, recording and site restoration) and completed the tasks in the following order:

- Search the area using a pre-arranged search pattern;

- Deploy a DSMB for marking the location;

- Take photos or video for an overview and details of the site;

- Put the metallic poles, using the sledgehammer, approximately every $2 \mathrm{~m}$ to describe the site;

- Deploy the retractable reel to set up a grid over the site;

- Sketch up the site in accordance to the grid and keep notes on the blank slate; 
- Use the depth meter to measure the depth at the edge of each pole;

- Use the plastic measurement tape (and the plummet) to measure slope (and horizontal) distances between the poles;

- Use the blank slate with the sketch of the site to write down the depth readings and the measured horizontal distances;

- Use the underwater photo/video camera to take two stripes of a series of almost vertical overlapping frames, following a U-pattern over the site (the sequential frames overlap approximately more than $60 \%$ and the stripes more than $30 \%$ );

- Retract the reel, remove the metallic poles and remove the DSMB from the site.

\subsection{Post-Dive Procedures}

After ascent and the proper care of personal, scuba equipment has followed the post-dive procedures including a debriefing of the dive, downloading the photo/video files from camera, copying the sketches and the measurements and finally, logging the dive and writing a report for the dive.

\section{Data Processing}

The processing of recorded data in favor of creating a 3D model is an optional module of the course, which is provided at those divers that have a special interest in mapping and 3D representations.

After the "minidive", a workshop has been organized in order to process the recorded data and produce a 3D model of the training site. The processing has been performed following the bellow described steps (Table 1), as refered in the manual of Agisoft Photoscan [16].

\subsection{Estimation of Image Quality}

Agisoft Photoscan provides the user with the ability to estimate image quality in order to avoid bad alignment's results due to vague images. Agisoft points all these images that their quality value is less than 0.5 units. The suggestion of the software is to remove these images and continue the photogrammetric processing with the rest of them for better and more accurate products. The quality value for GoPro images ranges between 0.86 units and 0.45 units and for Rollei images from 0.87 units to 0.60 units. Images with a value for the image quality less than 0.5 have been excluded. That is, nineteen images remained (one excluded) from the Gopro camera and all images from the Rollei remained for the processing.

\subsection{Photo Alignment}

Agisoft Photoscan Pro aligns the photos by matching the common points between overlapping images automatically (Fig. 1). Additionally, the software estimates for each photo the camera positions. Hence, it builds the sparse cloud model and eventually carries out the relative orientation. As regards the parameters of the command "align", high accuracy helps to obtain even more accurate camera position estimates. Therefore, disabled pair preselection means that each image is aligned with all the other images. Key point limit is the maximum number of image points that are taken into consideration in the stage. On the other hand, tie point limits is the maximum number of matching points for each image of the set. Photo alignment has been done in two chunks one for each data set (GoPro and Rollei). The parameters that have been set for the alignment was: accuracy-high, pair preselection-disabled, key point limit-40,000, tie point limit-10,000, which resulted in 37,536 tie points and 65,156 tie points for Gopro and for Rollei cameras respectively.

\subsection{Point Filtering}

Sometimes during the monitoring of the surface of the model the user may notice some points that deviate from the surface. This phenomenon is due to the small baseline of the immediate images. Hence, it is essential that this noise to be removed by deleting 
Table 1 The parameters during the stages of data processing (for GoPro and Rollei).

\begin{tabular}{|l|l|l|}
\hline Stage of data processing & $\begin{array}{l}\text { Data set 1 } \\
\text { (GoPro) }\end{array}$ & Data set 2 (Rollei) \\
\hline $\begin{array}{l}\text { Estimation of image quality } \\
(\text { accepted quality }>0.50)\end{array}$ & $\begin{array}{l}0.86-0.45 \\
(19 / 20)\end{array}$ & $\begin{array}{l}0.87-0.60 \\
(96 / 96)\end{array}$ \\
\hline Photo alignment (tie points) & 37,536 & 65,156 \\
\hline $\begin{array}{l}\text { Point filtering } \\
\text { (remaining tie points) }\end{array}$ & $\begin{array}{l}\text { no filtering } \\
31,516 \\
\text { (visible on 2 images each) }\end{array}$ & $\begin{array}{l}\text { 1st filtering 42,572 } \\
\text { 2nd filtering 26,278 }\end{array}$ \\
\hline Dense point cloud (number of points) & $\begin{array}{l}13,115,252 \\
22,460,317\end{array}$ \\
\hline Chunks alignment and merging & $\begin{array}{l}\text { Method: point based } \\
\text { Accuracy: high } \\
\text { Point limit: 40,000 }\end{array}$ & $\begin{array}{l}\text { Surface type: height field source data: dense cloud polygon count: 7,076,532 } \\
\text { interpolation: enabled }\end{array}$ \\
\hline Mesh building & $\begin{array}{l}\text { Blending mode: mosaic texture size: 4,096 } \\
\text { color correction: enabled }\end{array}$ \\
\hline Model texture building & $\begin{array}{l}\text { 6 scale bars } \\
\text { (measured distances } \\
\text { between 10 points) }\end{array}$ \\
\hline Camera alignment optimization &
\end{tabular}

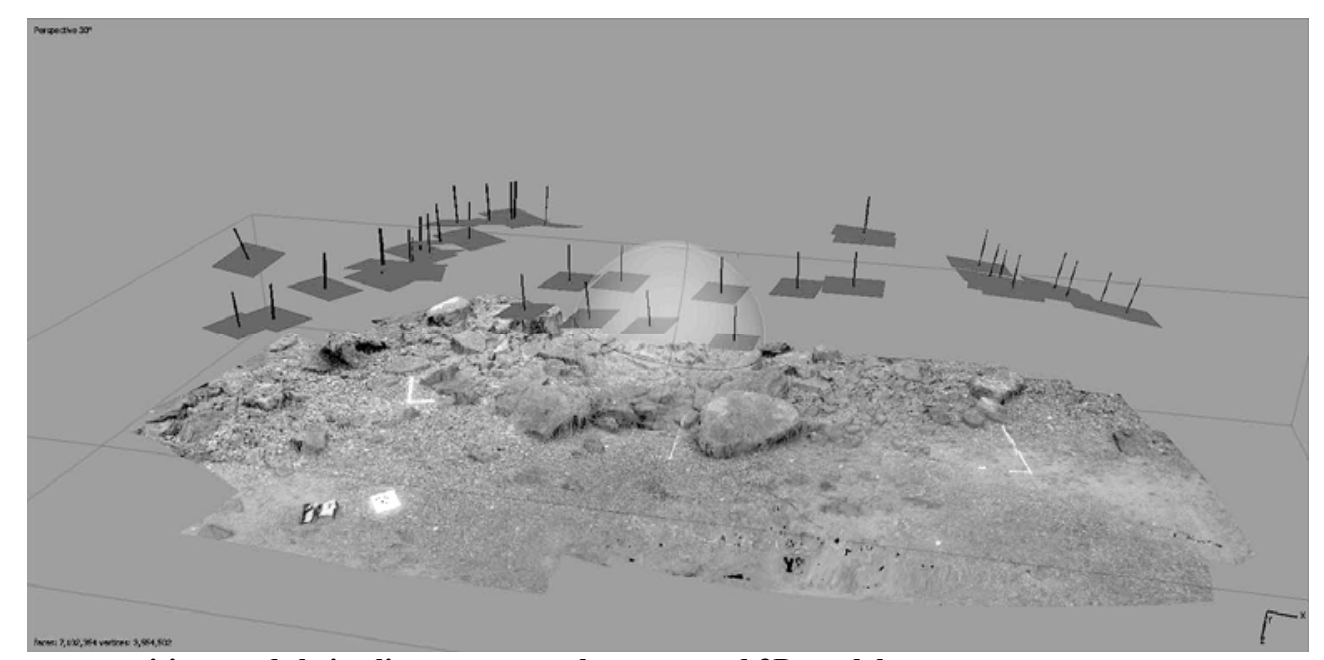

Fig. 1 The camera positions and their alignment over the generated 3D model texture.

these points in order to construct a better point cloud.

For the GoPro, there was no point filtering because 31,516 points out of the 37,536 are visible only on two images, obviously, because of the small overlapping between the recorded images. In this case, filtering has not been applied. The Rollei has been set to take sequences of three images whenever the shutter was released, which have created a lot of noise in the point cloud. An initial filtering, for keeping those points that have been visible on more than six images, resulted in 42,572 tie points. A consequent filtering with the parameters for the reconstruction uncertainty equal to 11 and for the reprojection error equal to 2 which have finally left 26,278 tie points for the rest of the processing.

\subsection{Dense Point Cloud}

Dense point cloud is constructed according to the estimated camera positions. Agisoft Photoscan produces depth information for each camera during the creation of the dense point cloud of the model. The product of the processing is combined into a single dense cloud of points. Agisoft recommends if the area to be reconstructed does not contain meaningful small details, then it is reasonable to chose aggressive depth filtering mode to sort out most of the outliers. Depth 
filtering can be Disabled, but this option is not recommended as the resulting dense cloud could be extremely noisy. Thus, small details like the rope and the metallic poles will not be reconstructed. The dense point cloud for the Gopro have included 13,115,252 points, whereas, the one for the Rollei 22,460,317 points, setting the reconstruction parameters for the quality to high and for the depth filtering mode to aggressive.

\subsection{Chunks Alignment and Merging}

When it is hard to construct a 3D model of whole scene in one go, chunks are used. Each chunk includes a part of the total images. In order to complete the creation of the 3D model, it is essential that the chunks be merged together. For merging the chunks, their alignment should be done in advance. In this project, point based method has been used for the alignment. This method aligns the chunks by matching images across both data sets. On the other hand, high accuracy leads to better and more accurate results. Additionally, point limit determines the maximum number of feature points that are taken into consideration in the alignment of the chunks. The aligning chunks parameters have been: method-point based, accuracy-high, point limit-40,000.

\subsection{Mesh Building}

Simple point clouds usually have not the appropriate characteristics in order to be used as the finally 3D model. Because of this, point clouds are converted to mesh clouds (polygon or triangle mesh models). With enabled (by default) interpolation mode, Photoscan will interpolate some surface areas within a circle of a certain radius around every dense cloud point. As a result, some holes can be automatically covered. Enabled setting is recommended for orthophoto generation. The following reconstruction parameters have been used: surface type-height field, source data-dense cloud, polygon count-high (7,076,532), interpolation-enabled.

\subsection{Model Texture Building}

In order to generate texture to the 3D model of the object, the building model texture command of Agisoft Photoscan software is applied. As texture mapping mode, Orthophoto is selected. Orthophoto mapping mode creates a compact texture surface whose generation is based on the orthographic projection. The selection of the texture generation parameter blending mode Mosaic uses the most proper photo and leads to more qualitative orthophotos and texture atlas. Texture size determines the size of texture atlas in pixel units. Finally, color correction is enabled, hence, the user can process data with an extent field of different brightness values. The texture mapping mode has been set to Orthophoto, with the following texture generation parameters: blending mode-mosaic, texture size-4,096, color correction-enabled.

\subsection{Camera Alignment Optimization}

During the alignment process, internal and external camera orientation parameters are calculated. In order to find these parameters, Agisoft Photoscan uses only image data. This process can lead to errors and specifically to some non-linear deformations of the generated model. The accuracy of this process is affected by some factors like the bad overlap between the images. So, the user needs to optimize camera alignment and Agisoft gives this ability. Hence, in this project, scale bar based optimization was used. Scale bar is a representation of any known distance in the field (Fig. 2). In this specific case study, six scale bars were used as reference distances (on a folding plastic ruler and on a carpenter's square) for the camera alignment optimization (Table 2).

\section{Accuracy Estimation}

The calculation of the quality value, before data processing, has shown a good quality for the two datasets of images. That is, it has been expected to produce an accurate 3D model. 


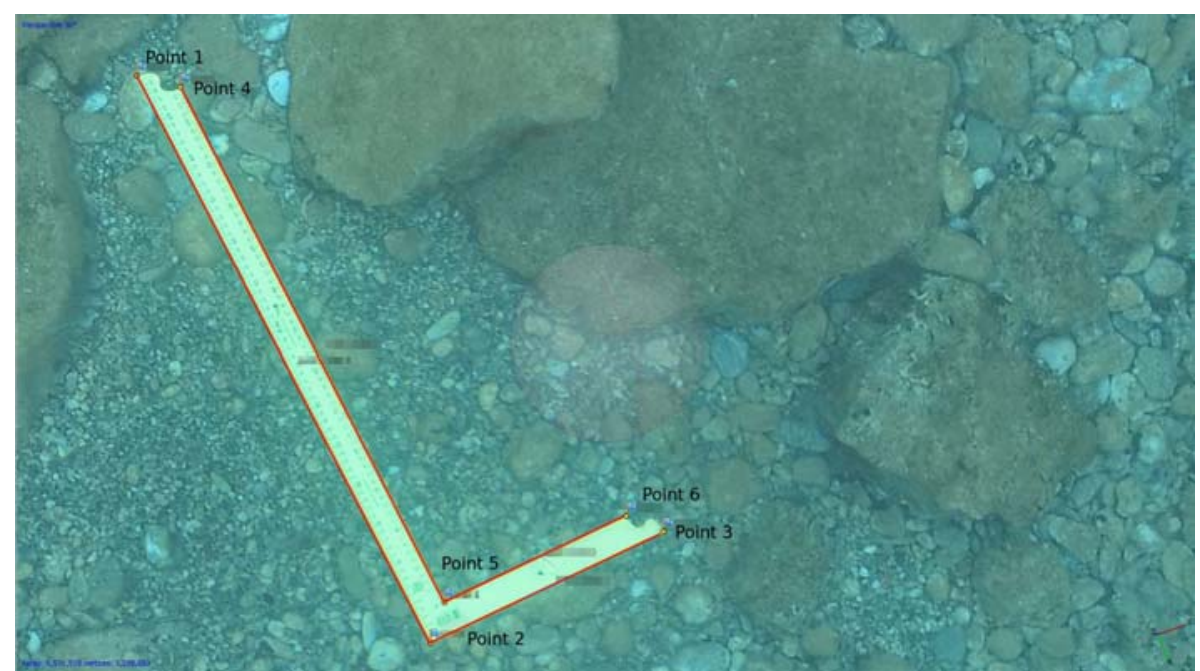

Fig. 2 View from the texture model, showing the reference distances between points 1-2, 2-3, 4-5 and 5-6, for the scale bar based optimization, it has been used a carpenter's square and a folding plastic ruler (not shown here).

Table 2 Comparison between the reference distances and the estimated ones by Agisoft Photoscan.

\begin{tabular}{lll}
\hline Scale bar & Reference distance $(\mathrm{m})$ & Estimated distance $(\mathrm{m})$ \\
\hline Points 1-2 & 0.700 & 0.700 \\
Points 2-3 & 0.295 & 0.295 \\
Points 4-5 & 0.630 & 0.635 \\
Points 5-6 & 0.200 & 0.229 \\
Points 7-8 & 0.200 & 0.172 \\
Points 9-10 & 0.800 & 0.800 \\
\hline
\end{tabular}

Table 3 Comparison between the sample distances and the calculated ones by Agisoft Photoscan.

\begin{tabular}{lll}
\hline Scale bar & Sample distance $(\mathrm{m})$ & Calculated distance $(\mathrm{m})$ \\
\hline Points11-12 & 0.800 & 0.801 \\
Points 13-14 & 0.200 & 0.169 \\
Points 15-16 & 0.310 & 0.308 \\
Points 15-18 & 0.230 & 0.230 \\
Points 16-17 & 0.230 & 0.229 \\
Points 17-18 & 0.310 & 0.308 \\
\hline
\end{tabular}

For the accuracy estimation of the final product, a second folding plastic ruler (deployed at right angle, with lengths of $80 \mathrm{~cm}$ and $20 \mathrm{~cm}$ on two perpendicular parts) and a measured blank slate $(23 \mathrm{~cm} \times 31 \mathrm{~cm})$ have been used as sample distances, positioned within the limits of the study area. Table 3 compares the sample distances, which have been recorded on the images during the underwater survey with the estimation distances, which have been calculated by Agisoft Photoscan after the creation of the 3D model.

Although the sample of distances that have been measured is very small and could not have been used for statistical analysis, it is indicative for the accuracy of the produced model. Most comparisons, have shown differences of less than $1 \mathrm{~cm}$ and just one sample distance have been calculated $3.1 \mathrm{~cm}$ smaller than the actual (between points 13 and 14). As a conclusion, this is considered a good result, in terms of accuracy, for the produced 3D model.

\section{Summary}

The outline for the PADI Underwater Survey Diver distinctive specialty course, intends to establish a protocol for the underwater recording and the 3D 
modeling of a small part at a diving site. The planning and the implementation of such an underwater survey in the framework of a training session and the data processing during a workshop have showed the simplicity in the application of the developed knowledge and skills, as well as the effectiveness of the course.

Although the course itself remains a basic introduction in underwater surveying, the inclusion of a workshop for the processing of the data has added educational value. Participants become the main performers of a complete underwater recording and $3 \mathrm{D}$ modeling project, from the planning and the implementation of the dive survey, to the recording and the processing of data and finally to the presentation of the produced 3D model in this paper.

During the training, knowledge from the fields of surveying and photogrammetry has been directly connected with diving skills. The perspective of this attempt is to incorporate the PADI Underwater Survey Diver distinctive specialty course in educational programs of academic institutions or to be accreditated as a vocational training one. In this context, it is expected to extend the curriculum for the knowledge development and cover more specialized subjects related to the underwater environment such as geology, oceanology, marine biology, maritime archaeology, underwater engineering or constructions etc..

\section{Acknowledgments}

The authors would like to thank the participants of the course, Chrystostomos Apostolou, Ines Cherif, Konstantinos Danis and Marios Giannourakos, who recorded the data during their confined water training. Additionally, is acknowledged Skopelos Dive Center which has organized the PADI Underwater Survey Diver distinctive specialty course and has provided the scuba equipment, the training material and the facilities for the preparation of the dive and the processing of data.

\section{References}

[1] Menna, F., Nocerino, E., Del, Pizzo, S., Bruno, F., and Remondino, F. 2015. "Underwater 3D Recording and Modeling.” Accessed October 15, 2015. http://www.int-arch-photogramm-remote-sens-spatial-infsci.net/XL-5-W5/index.html.

[2] ISPRS (International Society for Photogrammetry and Remote Sensing). 2015. "Underwater 3D Recording and Modeling.” ISPRS/CIPA Workshop. Accessed October 27, 2015. http://3dom.fbk.eu/files/underwater/index.html.

[3] Karara, H. M. 1989. Non-topographic Photogrammetry, (Second Edition). VA: American Society for Photogrammetry and Remote Sensing.

[4] Balletti, C., Beltrame, C., Costa, E., Guerra, F., and Vernier, P. 2015. "Underwater Photogrammetry and 3D Reconstruction of Marble Cargos Shipwreck.” In Int. Arch. Photogramm Remote Sens. Spatial Inf. Sci., XL-5/W5, 7-13.

[5] Bandiera, A., Alfonso, C., and Auriemma, R., 2015. "Active and Passive 3D Imaging Technologies Applied to Waterlogged Wooden Artifacts from Shipwrecks.” In Int. Arch. Photogramm Remote Sens. Spatial Inf. Sci., XL-5/W5, 15-23.

[6] Repola, L., Memmolo, R., and Signoretti, D. 2015. "Instruments and Methodologies for the Underwater Tridimensional Digitization and Data Musealization.” In Int. Arch. Photogramm Remote Sens. Spatial Inf. Sci., XL-5/W5, 183-190.

[7] Ricci, S., Antonelli, F., and Sacco, Perasso, C. 2015. "Aspects of Biodeterioration of Lapideous Submerged Artefacts: 3D Methodologies Application.” In Int. Arch. Photogramm Remote Sens. Spatial Inf. Sci., XL-5/W5, 191-194.

[8] D’Amelio, S., Maggio, V., and Villa, B. 2015. “3D Modeling for Underwater Archaeological Documentation: Metric Verifications.” In Int. Arch. Photogramm Remote Sens. Spatial Inf. Sci., XL-5/W5, 73-77.

[9] Bucci, G. 2015. "Underwater Surveys in Palaeo-Watercourses (Ferrara District-Italy).” In Int. Arch. Photogramm Remote Sens. Spatial Inf. Sci., XL-5/W5, 55-60.

[10] Bruno, F., Lagudi, A., Gallo, A., Muzzupappa, M., Davidde Petriaggi, B., and Passaro, S. 2015. "3D Documentation of Archeological Remains in the Underwater Park of Baiae.” In Int. Arch. Photogramm Remote Sens. Spatial Inf. Sci., XL-5/W5, 41-46.

[11] Bruno, F., Marcotulli, C., Vannini, G., and Drap, P. 2015. "Underwater Photogrammetry Methods for a Peculiar Case-Study: San Domenico (Prato-Italy).” In Int. Arch. Photogramm Remote Sens. Spatial Inf. Sci., XL-5/W5, 171-176. 
[12] Rende, F. S., Irving, A. D., Lagudi, A., Bruno, F., Scalise, S., and Cappa, P. et al. 2015. "Pilot Application of 3D Underwater Imaging Techniques for Mapping Posidonia Oceanica (L.) Delile Meadows.” In Int. Arch. Photogramm Remote Sens. Spatial Inf. Sci., XL-5/W5, 177-181.

[13] Papadimitriou, K. 2015. "Course Outline for a Scuba Diving Speciality 'Underwater Survey Diver'." In Int. Arch. Photogramm Remote Sens. Spatial Inf. Sci., XL-5/W5, 161-166.

[14] PADI (Professional Association of Diving Instructors). 2015. "Get Academic Credit for Your PADI Education." Accessed October 27 , 2015.
http://www.padi.com/mypadi/uploadedFiles/13_Referenc es_-_Reading_Room/132_Topics_of_Interest/10390_PA DIAcademicCredit_0115_v106_WEB.pdf.

[15] PADI (Professional Association of Diving Instructors). 2015. “PADI Instructor Manual.” Accessed October 27, 2015.

http://www.padi.com/mypadi/uploadedFiles/24_Training _Essentials/247_Downloadable_DIM/English/79173_Inst ructor_Manual_0115_v2015_Web.pdf.

[16] Agisoft LLC. 2014. "Agisoft Photoscan User Manual: Professional Edition.” Accessed October 27, 2015. http://www.agisoft.com/pdf/photoscan-pro_1_1_en.pdf. 